\title{
Statistical Modelling and Optimization of Fermentation Medium for Lincomycin Production by Streptomyces lincolnensis Immobilized Cells
}

\author{
Nayera A.M. Abdelwahed ${ }^{1}$, Eman Zakaria Gomaa ${ }^{2}$, Amira A. Hassan ${ }^{1}$. \\ ${ }^{1}$ Chemistry of Natural and Microbial Products Department, Pharmaceutical Industries Division, National Research \\ Centre, Giza, Egypt; ${ }^{2}$ Department of Biological and Geological Sciences, Faculty of Education, Ain Shams \\ University, Roxy, Cairo, Egypt.
}

\begin{abstract}
Response surface methodology was used to optimize lincomycin production by Streptomyces lincolnensis NRRL ISP5355 in submerged fermentation. Screening of fermentation medium components to find their relative effect on lincomycin production was done using Plackett-Burman design. Malt extract, dextrin, soluble starch and $\left(\mathrm{NH}_{4}\right)_{2} \mathrm{SO}_{4}$ were the most significant nutrient influenced on lincomycin production. Central composite design was applied to determine optimal concentrations of these factors and the effect of their mutual interactions. The interaction between soluble starch and $\left(\mathrm{NH}_{4}\right)_{2} \mathrm{SO}_{4}$ was found to enhance the production, whereas malt extract and dextrin exhibited an influence independent from the other two factors. Using this statistical optimization method, maximum lincomycin concentration of $1345 \mu \mathrm{g} / \mathrm{ml}$ was obtained which represented a $40.5 \%$ increase in titer than that acquired from the non-optimized medium. This statistically optimized medium was employed for lincomycin production through immobilization of Streptomyces lincolnensis by adsorption on synthetic cotton fibers. Immobilization technique improved the concentration to $1350 \mu \mathrm{g} / \mathrm{ml}$ higher than that produced from free cells cultures and could be maintained for longer than 17 days in a repeated batch system.
\end{abstract}

Key words: Optimization, lincomycin, Streptomyces lincolnensis, response surface methodology, immobilization

\footnotetext{
*Author for correspondence: niarawahed@yahoo.com
} 


\section{INTRODUCTION}

Lincomycin is one of the most widely used antibiotic obtained from Streptomyces lincolnensis and belongs to lincosamides group and it is effective against Gram positive bacteria the causative pathogens of infectuous diseases in animals and humans ${ }^{1}$. It shows in vitro and in vivo activity comparable to that of erythromycin against staphylococci, streptococci, and diplococci ${ }^{2}$. It has also been used as an industrial preservative; for example for impregnating paper and fabrics and as bacteriostatic rinse for laundered clothes. In addition, it has been used to suppress the growth of sensitive organisms in plate assays and in other biological media ${ }^{3}$.Various efforts have been made to improve the concentration of lincomycin ${ }^{4}$. Fermentation medium optimization is very important for the advancement of fermentation processes ${ }^{5}$. Designing of suitable media components for cultivation have cosiderable effect on production. Products yield could be improved by the application of statistical experimental design techniques which will economize time and cost more than routine single factor optimization. Several researchers applied Plackett-Burman (PBD) to choose influencing factors on the products concentration in the fermentation medium composition. These effective factors could be discovered by applying response surface methodology through central composite design (CCD) ${ }^{6}$. Modern biotechnology supported the utilization of immobilized cells for studying microbial fermentation processes. Besides, economic advantages such as extension of metabolic activities, recycle of cells for manufacturing products, increasing cell concentration and preventing cells to be driven by high stream rates were applied ${ }^{7}$. Cell-immobilization was demonstrated to be useful in the production of cephamycin C or clavulanic acid by $S$. clavuligerus; neomycin by $S$. marinensis or $S$. fradiae $^{8}$. The present work deals with the production of lincomycin by Streptomyces lincolnensis NRRL ISP-5355 in a statistically optimized medium which in turn was used for production using immobilized cells adsorbed on synthetic cotton fibers.

\section{MATERIALS AND METHODS}

Microorganism and culture conditions

Streptomyces lincolnensis NRRL ISP-5355 obtained from the Northern Regional Research Laboratory culture collection (NRRL), Peoria, Illinois, USA was used in this study for lincomycin production and it was maintained on slopes containing agar medium composed of (g/l): starch, 20; $\mathrm{NaNO}_{3}, 2 ; \mathrm{K}_{2} \mathrm{HPO}_{4}, 0.5 ; \mathrm{MgSO}_{4} .7 \mathrm{H}_{2} \mathrm{O}, 0.5$ and agar 20. For inoculum preparation, cells from newly prepared slants were inoculated in $250 \mathrm{ml}$ Erlenmeyer flask containing $50 \mathrm{ml}$ starch-nitrate broth medium for $48 \mathrm{~h}$. The obtained seed culture was inoculated into the desired fermentation medium according to the design matrix with an inoculum size of $2 \mathrm{ml}$ adjusted to cell density of about $1.5 \times 10^{8}$ spores / ml. The initial $\mathrm{pH}$ was adjusted to 7.0 and fermentation cultures were incubated on a rotary shaker at $200 \mathrm{rpm}$ for 10 days at $30^{\circ} \mathrm{C}$. All the experiments were carried out independently and the results were the average of three replicate experiments.

\section{Estimation of lincomycin}

The resulting clear filtrate was used to determine its antibacterial activity by the agar diffusion method ${ }^{9}$ using Bacillus subtilis NRRL B-543 strain as the test organism. The inhibition zone diameters were measured after incubation of plates at $30^{\circ} \mathrm{C}$ for $24 \mathrm{~h}$ and lincomycin concentrations expressed as micrograms per ml were quantified 
from a standard curve constructed by plotting known concentrations of lincomycin (authentic sample from Sigma Aldrich) versus inhibition zone diameters.

\section{Optimization of lincomycin production Effect of different incubation times}

A modified fermentation media composition from previously cited report ${ }^{10}$ was employed containing (g/l): soluble starch 20, glucose 35, soyabean meal 20, corn steep liquor $10, \mathrm{~K}_{2} \mathrm{HPO}_{4} 0.4,\left(\mathrm{NH}_{4}\right)_{2} \mathrm{SO}_{4}$ 1. The medium was sterilized at $121{ }^{\circ} \mathrm{C}$ for $15 \mathrm{~min}$, inoculated and incubated for 10 days at $30^{\circ} \mathrm{C}$ with agitation speed $(200$ rpm) and $\mathrm{pH}$ 7.0. Cultures were removed every day of incubation and tested for lincomycin concentration.

\section{Screening for medium components using Plackett-Burman design}

PBD was adopted for the selection of significant media components which influence production of lincomycin by Streptomyces lincolnensis. A total of 10 chemical components at two levels, high $(+)$ and low (-) were involved in the 12 trials to determine their effects on lincomycin production (Table2). List media components, codes, and levels of the different variables of the experimental design were given in Table1. The effect of each variable on lincomycin production was calculated and their significance was determined via Student's t-test using Minitab 17.0 (trial version). The variables with confidence levels above $95 \%$ and higher absolute tvalue were considered to have significant effect on lincomycin production and chosen for further optimization by response surface methodology (RSM). The Plackett-Burman experimental design is based on the first order polynomial model which is as follows:

$\mathrm{Y}=\beta_{0}+\beta_{1} \mathrm{X}_{1}+\beta_{2} \mathrm{X}_{2}+\beta_{3} \mathrm{X}_{3}+\beta_{4} \mathrm{X}_{4}+\ldots \ldots \ldots \ldots+\beta_{10} \mathrm{X}_{10} \quad$ equation (1)

where $\mathrm{Y}$ is the predicted response (lincomycin concentration), $\beta_{0}$ is the constant coefficient , $\beta_{1}-\beta_{10}$ are linear coefficients, $X_{1}-X_{10}$ are the independent variables. The significance of each variable was determined via Student's t-test. Statistical analysis of the responses were performed and represented in Table 3.

\section{Optimization of significant media components}

The optimum levels of the major variables which were selected through PB design were determined by the central composite design (CCD) of the response surface methodology (RSM).The selected significant factors, codes and their levels used in the (CCD) experiment are given in Table 4. The rest of the factors were kept at a constant level following their requested concentrations from the main effect result. According to the CCD, 31 experimental runs were fitted to the following second order polynomial model:

$$
\begin{aligned}
& Y=\beta_{0}+\beta_{1} X_{1}+\beta_{2} X_{2}+\beta_{3} X_{3}+\beta_{4} X_{4}+\beta_{11} X_{12}+\beta_{22} X_{22}+\beta_{33} X_{32}+\beta_{44} X_{42}+\beta_{12} X_{1} X_{2}+\beta_{13} X_{1} X_{3}+ \\
& +\beta_{14} X_{1} X_{4}+\beta_{23} X_{2} X_{3}+\beta_{24} X_{2} X_{4}+\beta_{34} X_{3} X_{4}
\end{aligned}
$$

Where $\mathrm{Y}$ is the dependent variable (lincomycin concentration), $\mathrm{X}_{1}, \mathrm{X}_{2}, \mathrm{X}_{3}, \mathrm{X}_{4}$ are the independent variables, $\beta_{0}$ is constant coefficient $\beta_{1}, \beta_{2}, \beta_{3}, \beta_{4}$ the linear coefficients, $\beta_{11}, \beta_{22}, \beta_{33}, \beta_{44}$ are the quadratic coefficients and $\beta_{12}, \beta_{13}, \beta_{14}, \beta_{23}, \beta_{24}, \beta_{34}$ are the interaction coefficients. The developed regression model was evaluated by analyzing the values of regression coefficients, analysis of variance (ANOVA), p- and Fvalues. The quality of fit of the polynomial model equation was expressed by the coefficient of determination, $\mathrm{R}^{2}$. The statistical software Minitab 17.0 was used to 
identify the experimental design as well as to generate a regression model to predict the optimum combinations considering the effects of linear, quadratic and interaction on lincomycin concentration. The two-dimensional (2D) contour plot is generally the graphical representation of the regression equation.

\section{Optimization of response and model validation}

The "Response Optimizer" option of the Minitab software was used to search for a combination of the factors involved that jointly optimize lincomycin production with the retention of a high viability. Desirability is a measure of how well the optimal solution satisfies the aim of the responses. A desirability of one indicates complete satisfaction while a desirability of zero indicates that the response is not acceptable.

\section{Preparation of immobilized cells}

Streptomyces lincolnensis NRRL ISP-5355 cells were immobilized on synthetic cotton fibers procured from the local market. A fixed weight $(0.5 \mathrm{~g})$ of this immobilization material was sterilized with $50 \mathrm{ml}$ of the previously optimized fermentation medium into each of the Erlenmeyer flasks $(250 \mathrm{ml})$. Seed culture of 48 hours growth was inoculated ( $2 \mathrm{ml} /$ flask) then incubated on a rotary shaker $200 \mathrm{rpm}$ at $30^{\circ} \mathrm{C}$. Repeated batch production of lincomycin from Streptomyces lincolnensis immobilized on synthetic cotton fibers was achieved by aseptic removal of fermentation medium at the end of fermentation time and was replaced with a fresh one. Each replacement of production medium was designated as a cycle of fermentation by reused cells for lincomycin production.

\section{RESULTS}

Production of lincomycin in batch cultivation In the batch cultivation, production of lincomycin started near $24 \mathrm{~h}$ of cultivation, still during the growth phase, most of the production occurred later, lincomycin was also increased by increasing the time of incubation period. Maximum production of $800 \mu \mathrm{g} / \mathrm{ml}$ was obtained at day $9(216 \mathrm{~h})$ of fermentation. The concentration remained constant during almost 2 days after that it began to decrease as shown in Fig.1.

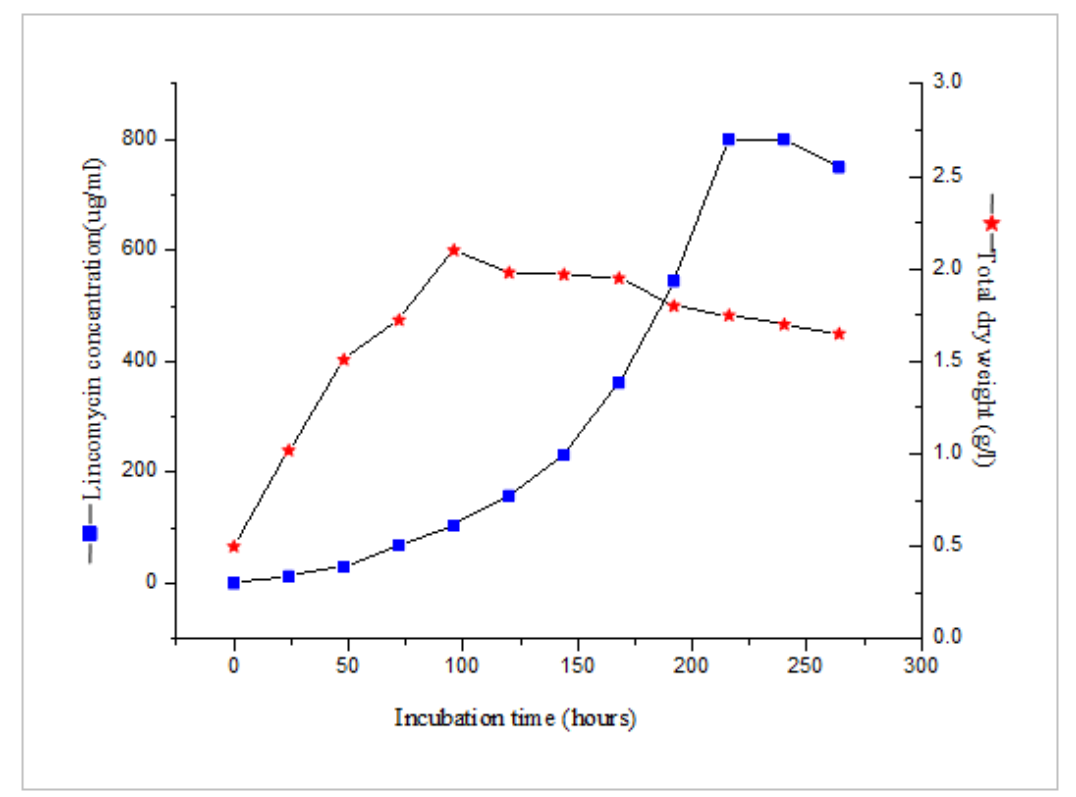

Fig. 1 - Effect of different incubation time on cell growth and lincomycin production 


\section{Optimization by PBD}

The importance of the 10 components, namely, malt extract, soyabean meal, corn steep liquor, dextrin, glycerol, soluble starch, $\mathrm{K}_{2} \mathrm{HPO}_{4}$, yeast extract, glucose and $\left(\mathrm{NH}_{4}\right)_{2} \mathrm{SO}_{4}$ for lincomycin production was investigated by PBD a statistical method aimed to screen by few experimental runs the most effective factors influenced on the production process for the improvement of lincomycin concentration ${ }^{11}$.

The main effect of each medium constituent on lincomycin concentration is presented in Fig. 2. The difference between the averages of measurements calculated at the high level $(+1)$ and at the low level $(-1)$ of each component estimated the main effect. The greater the deviation from the parallel ( $\mathrm{x}-$ axis), the greater is the magnitude of the main effect as indicated by the positive effect graphs of soluble starch and dextrin also the negative effect of malt extract and $\left(\mathrm{NH}_{4}\right)_{2} \mathrm{SO}_{4}$. When the main effect of a factor is positive, lincomycin production increases as the factor is changed from low to high level as showed in graphs of soyabean meal, corn steep liquor, $\mathrm{K}_{2} \mathrm{HPO}_{4}$ and glucose whereas for a negative main effect, the opposite behaviour (a decrease in the lincomycin production) is observed as proved in case of glycerol and yeast extract.

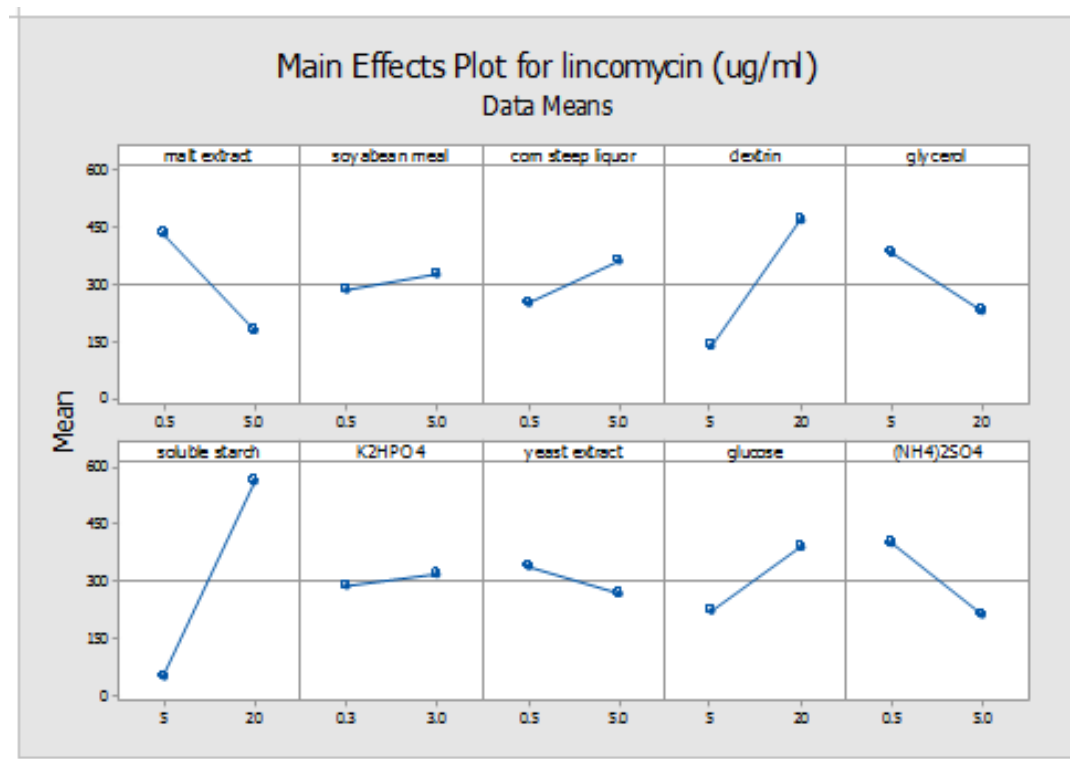

Figure 2- Effect of medium component factors on lincomycin production

The measure of impact of each factor has on the response in decreasing order was obviously illustrated by the Pareto chart which illustrates the order of significance of the variables (media components) influencing lincomycin production in PlackettBurman design. The statistically significant factors indicated by their long columns are crossed by a line confirmed their large influence on the production ${ }^{12}$. Among the 10 variables, the linear effect of soluble starch demonstrated the better significance by $45.726 \%$ followed by dextrin, malt extract and $\left(\mathrm{NH}_{4}\right)_{2} \mathrm{SO}_{4}$ showed effects of $29.183 \%, 2.486 \%$ and $17.07 \%$ respectively. Whereas the less significant factors have (standardized effect smaller than 15.89) at 96\% confidence level $(\alpha=0.04)$. As shown in Fig. 3, soluble starch, dextrin, malt extract, and $\left(\mathrm{NH}_{4}\right)_{2} \mathrm{SO}_{4}$ were the most significant factors (standardized effect is greater than 15.89) at $96 \%$ confidence level $(\alpha=0.04)$. 


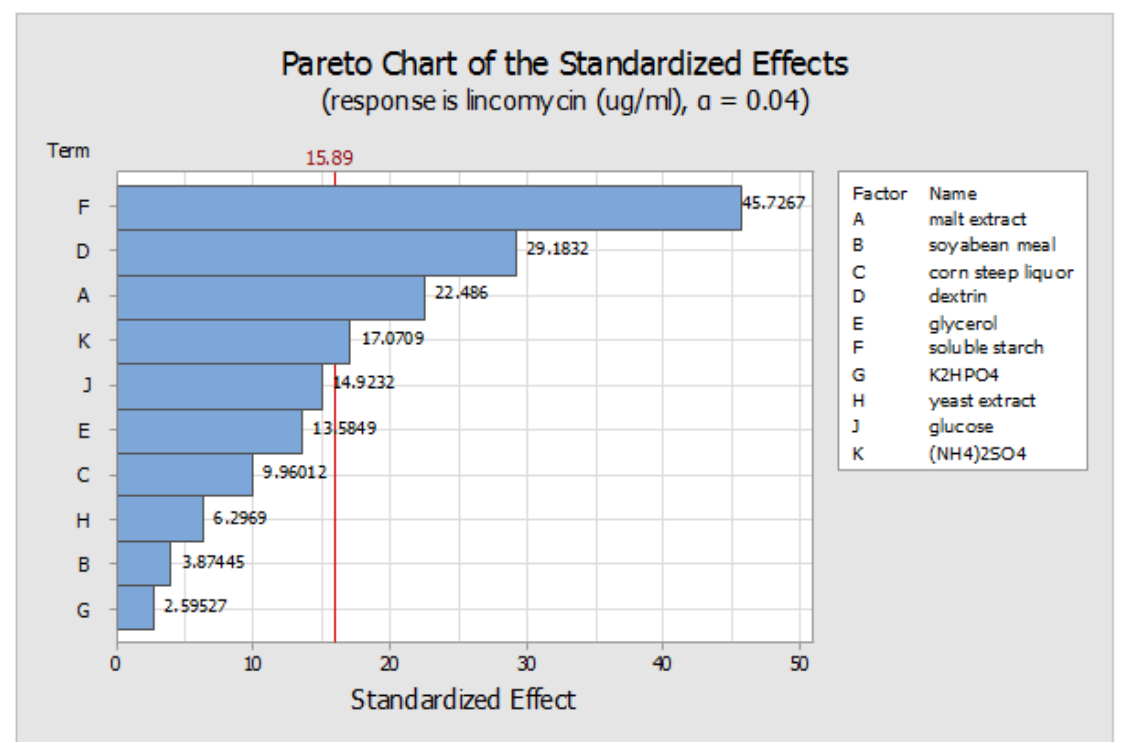

Figure 3- Pareto chart of absolute t-value for coefficients

Based on the statistical analysis, the effects of soluble starch, dextrin, malt extract and $\left(\mathrm{NH}_{4}\right)_{2} \mathrm{SO}_{4}$ had confidence levels above $95 \%$ with a probability value less than 0.05 and corresponding to higher absolute t- value were considered to have significant influence on the production and were selected for further optimization by response surface methodology. The coefficients of determination are high $\left(\mathrm{R}^{2}=\right.$ $99.98 \%$ and adjusted $\mathrm{R}^{2}=99.74 \%$ confirmed the explanation of more than $99 \%$ of total variability in the response derived by the first-order polynomial predicted equation given as follows:

(Y)lincomycin $(\mathrm{ug} / \mathrm{ml}) \quad=\quad$-209.5- 56.38 malt extract +9.71 soyabean meal+ 24.97 corn steep liquor +21.952 dextrin -10.219 glycerol +34.397 soluble starch. $+10.85 \mathrm{~K}_{2} \mathrm{HPO}_{4}-15.79$ yeast extract+ 11.226 glucose $-42.80\left(\mathrm{NH}_{4}\right)_{2} \mathrm{SO}_{4}$

equation (3)

The (CCD) under the (RSM) help the researcher in explaining the relationship between the response and the factors affecting the response therefore could in our study demonstrating the optimal concentrations of the most significant independent variables enhancing lincomycin production ${ }^{13}$. Based on the $\mathrm{PBD}$, four factors malt extract $\left(\mathrm{X}_{1}\right)$ dextrin $\left(\mathrm{X}_{4}\right)$, soluble starch $\left(\mathrm{X}_{6}\right)$ and $\left(\mathrm{NH}_{4}\right)_{2} \mathrm{SO}_{4}\left(\mathrm{X}_{10}\right)$ were selected. Optimization of process condition was performed according to the design of experiment as shown in Table 4. Lincomycin concentration attained its maximum value of $1340 \mu \mathrm{g} / \mathrm{ml}$ in run number 23 in which ammonium sulphate was used in its lowest concentration, while the minimum antibiotic titer $(1048 \mu \mathrm{g} / \mathrm{ml})$ was detected in run number 17 where starch was added in its minimum level.

The multiple regression analysis was applied on the data in Table 4 and the attained second-order polynomial equation could well explain the relationship between lincomycin concentration $(\mathrm{Y})$ and the four test variables in uncoded units:

Y(lincomycin conc. ug/ml $)=927-290.3$ malt extract- 7.99 dextrin+ 37.62 soluble starch- $22.8\left(\mathrm{NH}_{4}\right)_{2} \mathrm{SO}_{4^{-}} 26.7$ malt extract*malt extract+ 0.104 dextrin *dextrin- 0.548 soluble starch*soluble starch $+69.9\left(\mathrm{NH}_{4}\right)_{2} \mathrm{SO}_{4} *\left(\mathrm{NH}_{4}\right)_{2} \mathrm{SO}_{4}+$ +12.06 malt extract* dextrin -1.08 malt extract* soluble starch -10.7 malt extract * $\left(\mathrm{NH}_{4}\right)_{2} \mathrm{SO}_{4}-0.133$ dextrin* soluble starch -2.79 dextrin* $\left(\mathrm{NH}_{4}\right)_{2} \mathrm{SO}_{4} \quad-1.45$ soluble starch* $\left(\mathrm{NH}_{4}\right)_{2} \mathrm{SO}_{4}$ equation (4) 
The regression coefficients, along with the corresponding t-value and p- values, for the model of lincomycin production by Streptomyces lincolnensis are presented in Table 5. The coefficient corresponding to larger absolute t-value and smaller $\mathrm{p}$-value was considered as the more significant one ${ }^{14}$. Significance of coefficients has been reported to be directly proportional to the t-value and inversely to $p$-value ${ }^{15}$. The terms and their corresponding p-values recommend that the independent variables malt extract and soluble starch have significantly affect lincomycin production. These suggest that their concentrations have a direct relationship with the antibiotic activity in the fermentation medium. The significance of positive coefficient for soluble starch indicated a linear effect to increase lincomycin production, while negative coefficient for malt extract was seen to diminish the production. The quadric term of soluble starch and $\left(\mathrm{NH}_{4}\right)_{2} \mathrm{SO}_{4}$ are more significant than the other quadratic terms $(\mathrm{p}<0.001)$, indicate that it can perform as limiting components and any change in their concentrations will change the antibiotic concentration .Also, the interactions effect between malt extract and dextrin has significant effects on antibiotic activity $(\mathrm{P}<0.05)$. In this case, malt extract, soluble starch, (soluble starch $)^{2},\left(\left(\mathrm{NH}_{4}\right)_{2} \mathrm{SO}_{4}\right)^{2}$ and malt extract *dextrin are significant model terms. The polynomial equation was further tried for adequacy by ANOVA, which is used to determine whether there are any significant differences between the means of the multiple independent variables. The ANOVA of the quadratic regression model showed that the model is significant, as it can be seen from the Fisher's F-test (F model $=13.34)$ with a low probability value $(\mathrm{p}=0.000)$. A good coincidence between the polynomial regression model and experimental data, with a coefficient of determination being $\left(\mathrm{R}^{2}=92.11 \%\right)$ proposing that only about $7.89 \%$ of the total variations cannot be clarified by the model. The value of adjusted determination coefficient (Adjusted $\mathrm{R}^{2}=85.21 \%$ ) is also high, proposing a high significance of the model.

The two-dimensional (2D) contour plots are graphical representation used to investigate the interaction among variables (malt extract, dextrin, soluble starch and $\left(\mathrm{NH}_{4}\right)_{2} \mathrm{SO}_{4}$ and to determine the optimum concentration of each factor for maximum lincomycin production. The contour lines were plotted with data predicted from equation (4). Each contour curve represents an infinite number of combinations of two test variables with the other two variables maintained at their respective zero level. The shapes of contour plots indicate the nature and extent of the interactions. Significance of the interactions between the corresponding variables is indicated by an elliptical or saddle nature of the surface plots ${ }^{16}$. When moving along the major and minor axis of the ellipse, the optimal values of variables are obtained and the response at the centre point yields maximum lincomycin production .The maximum predicted value of lincomycin concentration is indicated by the surface restricted in the regular oval shape in the contour diagram. It was noticed that around $30 \mathrm{~g} / \mathrm{l}$ of soluble starch in its interaction with a low concentration of malt extract and high concentration of dextrin around $40 \mathrm{~g} / \mathrm{l}$ enhance lincomycin production as expressed by the dark green area (Fig. 4a, b). Whereas, interaction of low concentration of malt extract with high concentration of $\left(\mathrm{NH}_{4}\right)_{2} \mathrm{SO}_{4}$ around $2 \mathrm{~g} / \mathrm{l}$, improved lincomycin production (Fig. 4e). On the other hand, Fig. $4 \mathrm{~d}$ visualized an increase in lincomycin concentration with high concentration of dextrin around $(40 \mathrm{~g} / \mathrm{l})$ when interacted with low concentration of $\left(\mathrm{NH}_{4}\right)_{2} \mathrm{SO}_{4}$. 


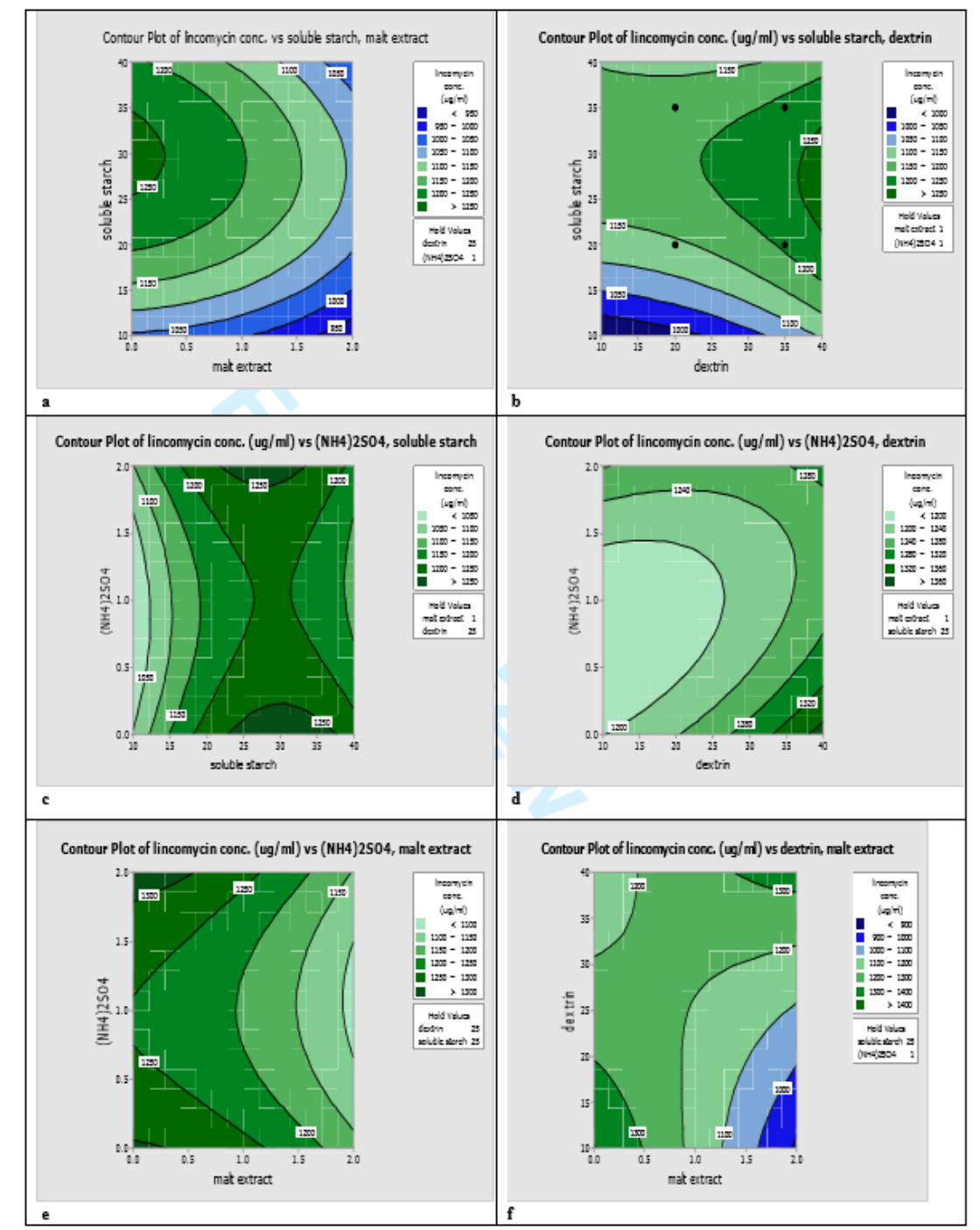

Figure 4- Contour plot showing the interaction among variables for maximum lincomycin production (a) soluble starch with malt extract, (b) soluble starch with dextrin, (c) $\left(\mathrm{NH}_{4}\right)_{2} \mathrm{SO}_{4}$ with soluble starch, (d) $\left(\mathrm{NH}_{4}\right)_{2} \mathrm{SO}_{4}$ : with dextrin, $\left(\mathrm{NH}_{4}\right)_{2} \mathrm{SO} 4$ with malt extract and (f) dextrin with malt extract . Each combination of two test variables with the other two variables maintained at their respective zero.

The response surfaces in Fig. $4 \mathrm{c}$ have a saddle behavior or minimax nature where the stationary point is not a minimum or maximum point but a saddle point. From this point near the center of the design, increasing or decreasing both variables concentrations at the same time leads to a decrease in the response. This kind of contour plot is peculiar, because the response (lincomycin concentration) approaches optimum and then moves away from it. While, from the same point increasing either variable while decreasing the other leads to an increase in the response. It can be observed that increasing soluble starch concentration and decreasing $\left(\mathrm{NH}_{4}\right)_{2} \mathrm{SO}_{4}$ concentration increased lincomycin concentration. Therefore, a negative effect of the interaction of these variables is apparent as predicted by equation (4). On the other hand, lower and higher levels of both did not result in higher lincomycin concentration. Similarly, Fig. 4a, b, d, e showed the negative effect of the interaction of soluble starch with malt extract, soluble starch with dextrin,$\left(\mathrm{NH}_{4}\right)_{2} \mathrm{SO}_{4}$ with dextrin and $\left(\mathrm{NH}_{4}\right)_{2} \mathrm{SO}_{4}$ with malt extract respectively.In case of the interaction between dextrin and malt extract (Fig. 4f), lower and higher levels of both resulted in 
higher lincomycin concentrations then a decrease in production beyond these interaction levels.

\section{Validation of the optimized medium component}

Eventually, in order to determine the optimal levels of each variable for maximizing lincomycin concentration, the application of desirability function method was applied after making the regression model, (Fig.5). Response optimization method involved a combination of all desired variable levels (d) into one measurement expressed by a single desirability index, D. When D was close to 1 indicating the fact that the function increases linearly towards the desired target values of lincomycin concentration ${ }^{17}$. In the recommended optimal model parametric settings of maximum lincomycin concentration predicted malt extract $0.5 \mathrm{~g} / \mathrm{l}$, dextrin $10 \mathrm{~g} / \mathrm{l}$, soluble starch $30.0 \mathrm{~g} / \mathrm{l}$ and $\left(\mathrm{NH}_{4}\right)_{2} \mathrm{SO}_{4} 2.0 \mathrm{~g} / \mathrm{l}$ were set. The response for these values for lincomycin production with desirability of 1.0000 was $1425.58 \mu \mathrm{g} / \mathrm{ml}$. Therefore, the predicted optimum condition was verified experimentally and compared with the predicted data. The measured lincomycin concentration was 1345 $\mu \mathrm{g} / \mathrm{ml}$ revealed more than $90 \%$ accuracy to the predicted concentration, indicating the model validation under the tested conditions.

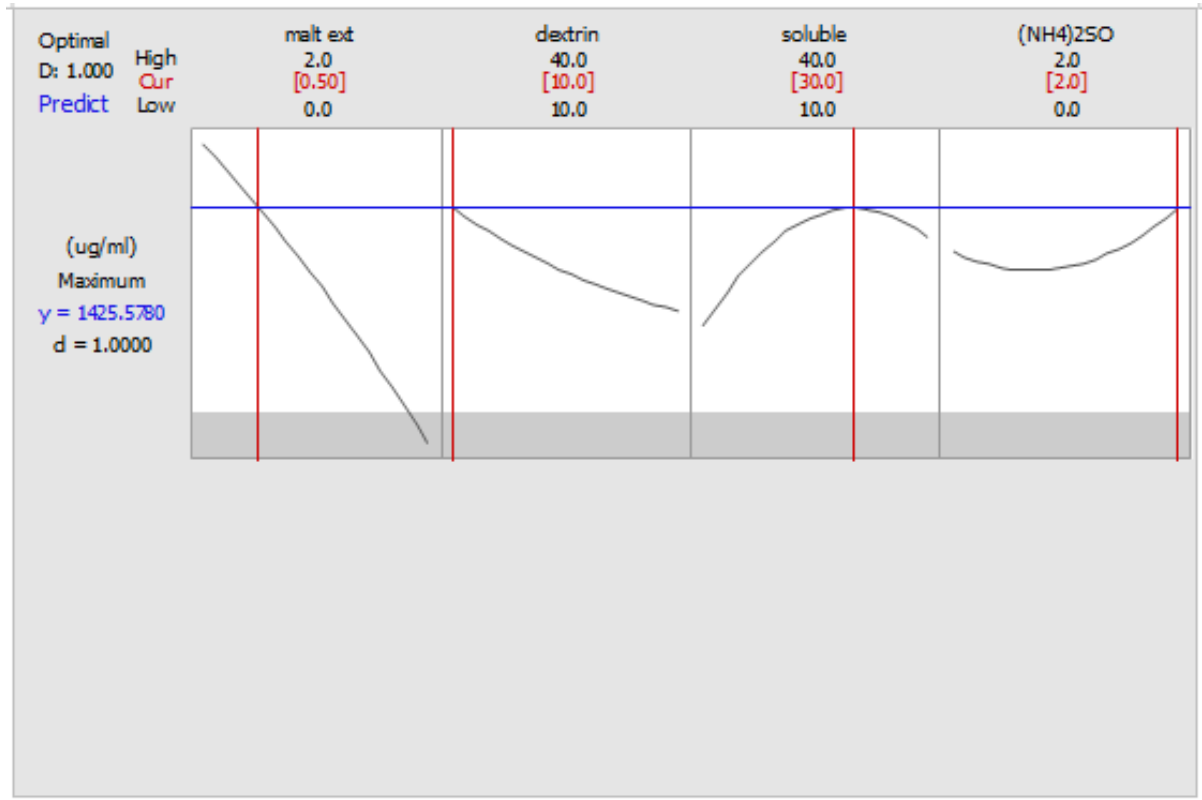

Figure 5- Recommended input variables to achieve maximum lincomycin concentration (ug/ml)

Use of the optimized medium for lincomycin production by immobilized cells A time course of lincomycin production with immobilized cells during $264 \mathrm{~h}$ fermentation was shown in Fig. 6. The antibiotic concentration from the immobilized cell culture was higher reached $1350(\mu \mathrm{g} / \mathrm{ml})$ at $192 \mathrm{~h}$ and remain constant whereas in the case of free cells a gradual decrease was detected from $1345(\mu \mathrm{g} / \mathrm{ml})$ to 1300 $(\mu \mathrm{g} / \mathrm{ml})$ after $192 \mathrm{~h}$. 


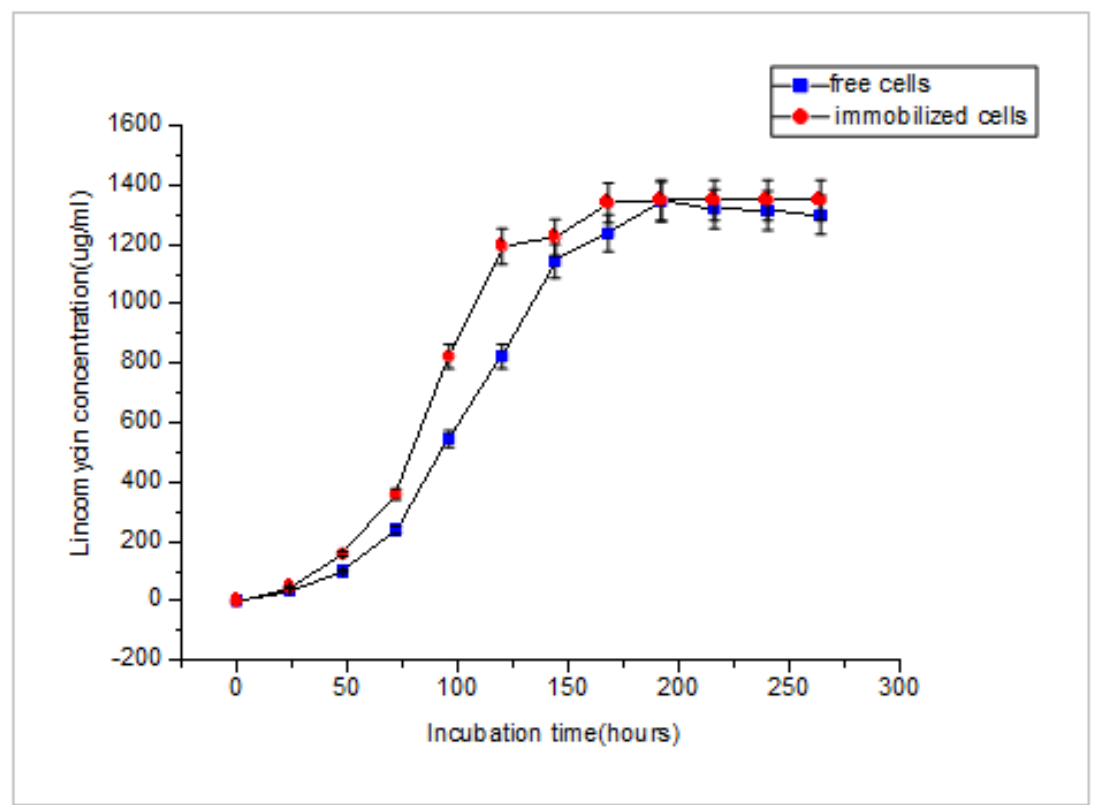

Figure 6- Effect of incubation time on lincomycin production by free and immobilized cells. Error bars represent the standard deviation from a mean of three replicates

Lincomycin production by Streptomyces lincolnensis adsorbed on synthetic cotton fibers was studied up to five reused cycles as shown in Fig. 7. From the data, it is clear that during the first reuse, the lincomycin production increased gradually with the increase of fermentation time and reached a maximum of $1350 \mu \mathrm{g} / \mathrm{ml}$ at the end of $192 \mathrm{~h}$. For the second reuse, the cells were already in the late exponential phase and hence a faster initiation of the lincomycin production $1365 \mu \mathrm{g} / \mathrm{ml}$ after $144 \mathrm{~h}$ was recorded and remain constant. In the third reuse, it was noticed that a slight decrease in antibiotic production reached $(1239 \mu \mathrm{g} / \mathrm{ml})$ at the end of fermentation period.

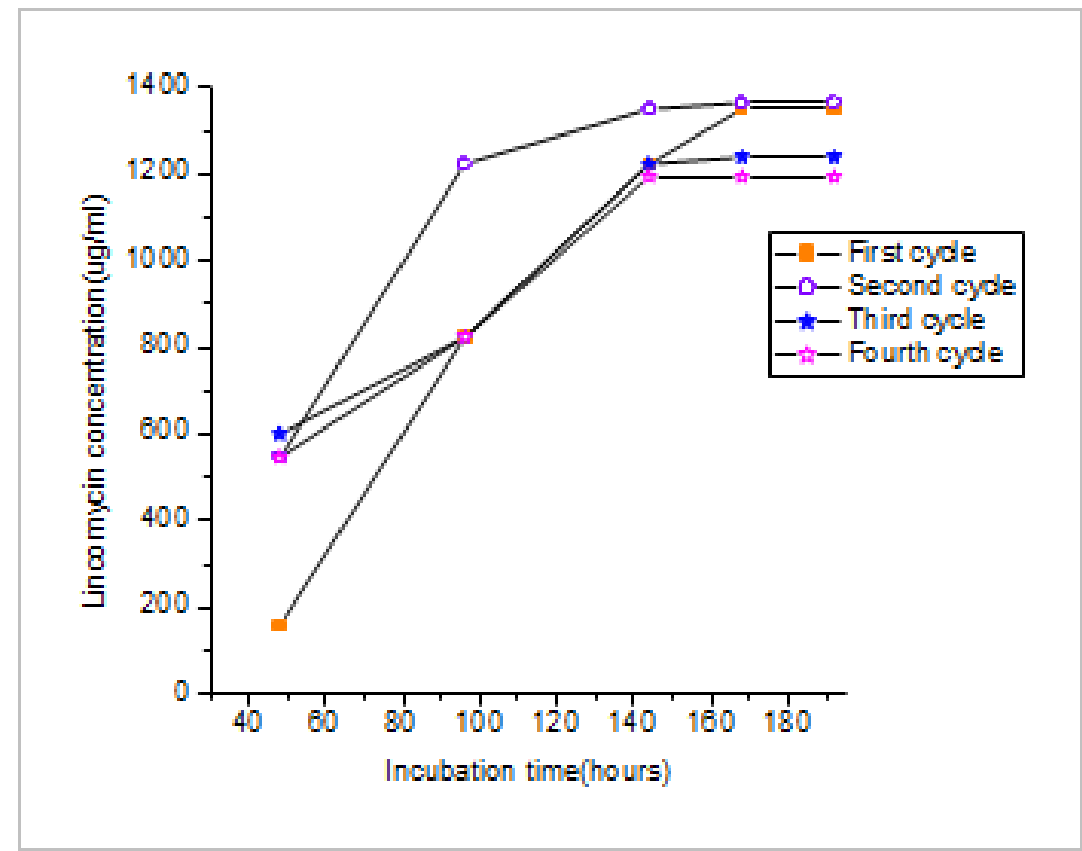

Figure 7-Effect of incubation time on lincomycin production by immobilized cells through repeated batch cycle 
Further decrease in lincomycin production was detected in the fourth and fifth reuse until it reached its minimum value of $824 \mu \mathrm{g} / \mathrm{ml}$.

Scanning electron micrographs showed that there are highly condensed but varying degrees of mycelial growth within synthetic cotton fibers (Fig. 8). A great advantage of synthetic fibers is that they are more durable than most natural fibers and do not break down easily when exposed to light, water, or oil. They made from synthesized polymers.

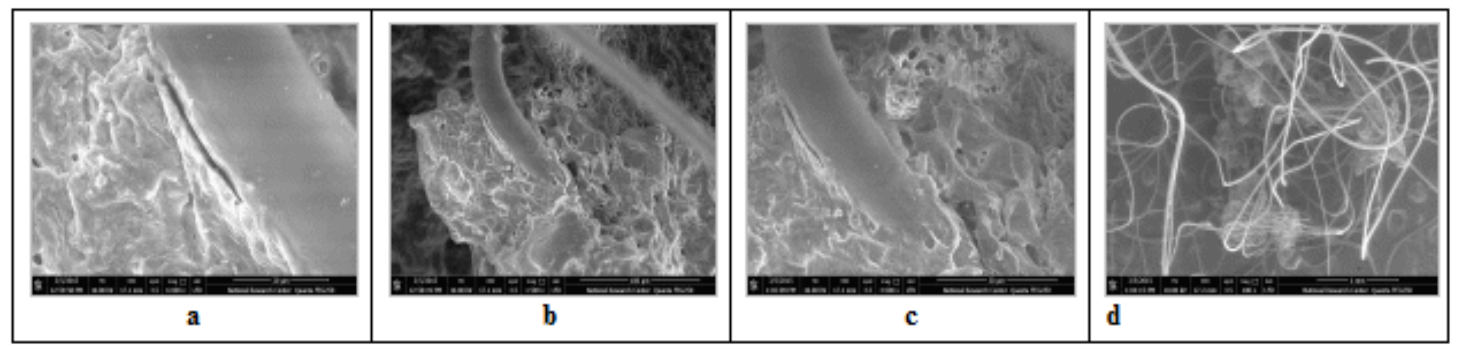

Figure 8- Scanning electron micrographs showing adhesion of Streptomyces lincolnensis adsorbed on synthetic cotton fibers: (a) Bar $=20 \mu \mathrm{m}$, (b) Bar $=100 \mu \mathrm{m}$, (c) Bar $=50 \mu \mathrm{m}$ and (d) Bar $=1 \mathrm{~mm}$.

\section{DISCUSSION}

The time course of the batch cultivation for lincomycin production revealed that cell growth occurred in $96 \mathrm{~h}$ associated with the beginning of production; however most of the production phase occurs with no association with the growth phase. This fact indicates that production is not associated with microorganism growth, in agreement with other results demonstrated the same behaviour ${ }^{18}$. Supplements and precursors provided in the culture usually reflect the role of the medium in the biosynthesis of antibiotics which generally depends on media composition and the function is considered in terms of what it gives to the culture ${ }^{19}$. Accordingly, screening of medium components for their significant effect on product formation in shake flask could be possible upon using statistical factorial designs like Plackett-Burman design (PBD). In this study, the predicted and observed responses along with design matrix presented in Table 2 revealed that the highest lincomycin concentration was found in run 7. This could be related to the presence of soluble starch, dextrin and organic nitrogen in high concentration level while run 3 showed the lowest lincomycin concentration where soluble starch and dextrin were existed in low concentration level despite the presence of glycerol at high level. One explanation for this may be due to the consumption of maximum quantity of soluble starch and glucose after the action of maximum cell growth formation. In this regard, many reports showed that the bio synthesis of antimicrobial agents depend on the composition of medium. Carbon source, like simple sugar in fermentation media enhanced growth as well as bioactive metabolite production rather than more complex carbons. Other finding revealed that the production of antibiotic by $S$. tanashiensis A2D was preferred in media containing soyabean meal as carbon source ${ }^{20}$. In view of significance of media components, soluble starch and dextrin as carbon sources, malt extract and $\left(\mathrm{NH}_{4}\right)_{2} \mathrm{SO}_{4}$ as organic and inorganic nitrogen source respectively were chosen as most significant ingredients in the fermentation medium for lincomycin production. A close relationship between the carbohydrate metabolism and the antibiotic production was discovered by carbohydrate utilization design. This finding has a certain parallel with the reports of other workers concerned with the nutrition of other strains or species ${ }^{21,22,23}$. Raytapadar and Paul ${ }^{24}$ described the production of antibiotic from Streptomyces aburaviensis 1DA-28 required starch as an important ingredient in the fermentation media, similar results were reported for the 
production of antimicrobial compound from Streptomyces sp. JAJ06 ${ }^{6}$. Zhu et al. ${ }^{25}$ gave an account on the importance of adjusting the concentration of $\left(\mathrm{NH}_{4}\right)_{2} \mathrm{SO}_{4}$ in fermentation media for the production of antibiotic by Streptomyces viridochromogenes. Starch concentration in the production medium is shown to influence production of antimicrobial substances by actinomycetes. In the present study, Streptomyces lincolnensis NRRL ISP-5355 was found to produce lincomycin to higher extent on increasing the starch concentration. Maximum bioactivity was observed at high starch concentration of 3\%. On the other hand, dextrin was the most significant carbon source in medium optimization for daptomycin antibiotic production by Streptomyces roseosporus NRRL $11379^{22}$ and tacrolimus production by Streptomyces tsukubaensis ${ }^{26}$. Other than carbon, nitrogen source is significant for the production of antibiotic substances. Supplementation of medium with organic as well as inorganic nitrogen source resulted in improvement of antibiotic production ${ }^{27}$. Our results were in agreement with Malt extract was the most significant organic nitrogen source for antitubercular pigmented antibiotic production from novel Streptomyces sp D25 ${ }^{28}$. Response surface methodology (RSM) is a mathematical statistical desing for the optimization of significant media components ${ }^{29}$. RSM gives adequate data for statistically acceptable result by less number of experimental runs ${ }^{30}$. RSM procedure with CCD mediated the enhancement of antibacterial compound production in several Streptomyces species: Streptomyces sindenensis, Streptomyces daufpe 3060, Streptomyces alboflavus $313^{31}$. In the present study, RSM was applied with CCD to optimize the levels of selected media components. This methodology favored $40.5 \%$ increase in lincomycin concentration by Streptomyces lincolnensis NRRL ISP-5355 compared with the non optimized medium. The repeated batch fermentations were conducted (every 192 hours) using immobilized cells in this optimized fermentation media containing $(\mathrm{g} / \mathrm{l})$ : malt extract 0.5 , dextrin 10 , soluble starch 30.0 and $\left(\mathrm{NH}_{4}\right)_{2} \mathrm{SO}_{4} 2.0$ as desired by the response optimization method along with soyabean meal 5 , corn steep liquor 5 , glycerol $5, \mathrm{~K}_{2} \mathrm{HPO}_{4} 3$, yeast extract 0.5 and glucose 20 as recommended by the main effect results from PBD. The use of synthetic cotton fibers to immobilize actinomycetes has already proven useful. It is comprised of manufactured fibers connecting with each other and has desirable properties such as high strength, high resistance to water and chemical degradation, with an open network of fibrous polymer matrix giving the potential for fast contact of immobilized cells with the surrounding watery medium. So the living cells adsorbed on it has great dispersion of suplement and oxygen exchange. The physical attachment of immobilized cells by adsorption makes an easy exchange of fermentation medium in the immobilized system for metabolic control or nutrient renewal .The effectiveness of maximum cell mass adsorption onto synthetic cotton fibers might be because of the formation of network by the matrices. The stability of the intracellular biosynthetic factors and activities of the secondary metabolic compounds provide evidence for supporting cell adhesion, proliferation and differentiation. Successful immobilization of filarnentous microorganisms on such open porous supports as sponge, polyurethane foam, cotton bags, nylon scrubbers or polystyrene has been reported before. Moreover, the synthetic cotton fibers adsorbed cultures showed the lowest viscosity, which could allow a better transfer and oxygen mixing. Hence, materials such as synthetic cotton fibers were selected for repeated batch fermentation ${ }^{32}$. Results obtained indicate that the immobilized cells on the synthetic cotton fibers led to a significant stability in the antibiotic activity and were able to maintain lincomycin at an almost constant high level making repeated batch production to be an attractive work. In another reports, glass wool harboring immobilized growing cells were reused and kept on producing oxytetracycline by S. rimosus and rifamycin B and SV by Amycolaptosis for 20 days 33. As otherwise mentioned, different researchers utilized cells adsorbed on different 
supporting matrices for various antibiotics production and reported higher antibiotic concentrations compared to that produced in free cells fermentation cultures ${ }^{34}$. The successful adsorption obtained may be due to an electrostatic attraction between Streptomyces cells and these artificial polymeric fibers.

\section{CONCLUSION}

As an added advantage the low price of artificial polymeric cotton fibers support, present some facility for the possibility of using adsorbed Streptomyces lincolnensis for lincomycin production. Further scale up and optimization studies are still necessary in order to apply the proposed study to commercial processes and to evaluate its techno-economical feasibility.

\section{ACKNOWLEDGMENTS}

This work was supported by National Research Center, Chemistry of Natural and Microbial Products Department, Pharmaceutical Industries Division (Egypt).

\section{REFERENCES}

1- Spizek J, Rezanka T. Lincomycin, clindamycin and their applications. Appl Microbiol Biotechnol. 2004; 64:455 - 464

2- Dousa M, Sikac Z, Halama M, Lemr K. HPLC determination of lincomycin in premixes and feedstuff with solid-phase extraction on HLB OASIS and LC-MS/MS confirmation. J of Pharm and Biomed Anal. 2006; 40: 981-986

3- Choi DB, Ryu SR, Choi OY, Yin P, Kwun KH. Recovery and purification of lincomycin from the culture broth of Streptomyces lincolnensis. J Ind Eng Chem. 2005; 11 (6): 932-937

4- Ye R, Wang Q, Zhou X. Lincomycin, rational selection of high producing strain and improved fermentation by amino acids supplementation. Bioprocess Biosyst Eng. 2009; 32 (4):521-529

5- Krouse MKD. Strategies for improving fermentation medium performance: a review. J Ind Microbiol Biotechnol. 1999; 23: 456-475

6- Jose PA, Sivakala K K, Jebakumar SRD.Formulation and statistical optimization of culture medium for improved production of antimicrobial compound by Streptomyces sp.JAJ06. Int.J Microbiol. 2013; 2013:1-9

7- Gautam P, Fatu A, Panday A, Szakasc G, Soccol CR. Microbial production of extracellular phytase using polystyrene as inert solid support. Bioresource Technol. 2002; 83: 229-233.

8- López-García MT, Rioseras B, Yagüe P, Álvarez JR, Manteca Á . Cell immobilization of Streptomyce coelicolor: effect on differentiation and actinorhodin production. Inter Microbiol .2014; 17 (2):75-80

9- Perez C, Pauli M, Bazevque P. An antibiotic assay by the agar well diffusion method. Acta Biol Med Exp. 1990; 15:113-115

10- Li XB, Zhao GR, Zheng H, Yuan YJ. Improved industrial fermentation of lincomycin by phosphorus feeding. Proc Biochem. 2007; 42 (4):662-668

11- Plackett R L, Burman JP.The design of optimum multifactorial experiments. Biometrika.1946; 33:305-325

12- Thella JS, Venugopal R (2011). Modeling of iron ore pelletization using $3^{* *(k-p)}$ factorial design of experiments and polynomial surface regression methodology. Powder Technol. 2011; 211:54-59

13- Box GEP , Wilson KB.On the Experimental Attainment of Optimum Conditions (with discussion). J R Stat Soc Series B. 1951; 13 (1):1- 45

14- Karthikeyan RS, Rakshit SK, Baradarajan A. Optimization of batch fermentation conditions for dextran production. Bioproc Eng. 1951; 15:247-251 
15- Akhnazarova S, Kafarov V. Experiment Optimization in Chemistry and Chemical Engineering, 1982, pp. 151-240. Moscow: MIR Publishers

16- Muralidhar RV, Chirumanila RR, Marchant R, Nigam P A response surface approach for the comparison of lipase production by Candida cylindracea using two different carbon sources. Biochem Eng J. 2001; 9:17-23

17- Deka D, Das SP, Sahoo N, Das D, Jawed M, Goyal D, Goyal A.Enhanced cellulase production from Bacillus subtilis by optimizing physical parameters for bioethanol production. ISRN Biotechnol. 2013; 2013: 1-11

18-Baptista-Neto A, Gouveia ER, Badino-Jr AC, Hokka CO. Phenomenological model of the clavulanic acid production process utilizing Streptomyces clavuligerus. Braz J Chem Eng. 2000; 17(04-07):809-818.

19-Elibol M. Optimization of medium composition for actinorhodin production by Streptomyces coelicolor A3(2) with response surface methodology. Process Biochem. 2004; 39:1057-1062

20- Singh LS, Sharma H, Talukdar NC. Production of potent antimicrobial agent by actinomycete, Streptomyces sannanensis strain SU118 isolated from phoomdi in Loktak Lake of Manipur, India. BMC Microbiol 2014; 14(1): 278.

21- Ruchi G, Anshu G, Khare SK. Lipase from solvent tolerant Pseudomonas aeruginosa strain: production optimization by response surface methodology and application. Bioresour Technol. 2008; 99:4796-4802

22- Ng IS, Ye C, Zhang Z, Lu Y, Jing K. Daptomycin antibiotic production processes in fedbatch fermentation by Streptomyces roseosporus NRRL 11379 with precursor effect and medium optimization. Bioproc Biosyst Eng. 2014; 37(3): 415-423

23- Himabindu M, Jetty A. Optimization of nutritional requirements for gentamicin production by Micromonospora echinospora. Ind J Exp Biol. 2006; 44:842-848

24- Raytapadar S , Paul AK . Production of an antifungal antibiotic by Streptomyces aburaviensis 1DA- 28. Microbiol Res . 2001; 155: 315-323

25- Zhu CH, Lu FP, He YN, Han ZL, Du LX. Regulation of avilamycin biosynthesis in Streptomyces viridochromogenes: effects of glucose, ammonium ion, and inorganic phosphate. Appl Microbiol Biotechnol. 2007; 73:1031-1038

26- Archana RT, Nishtha KS, Umesh L. Optimization of nutrient components for tacrolimus production by Streptomyces tsukubaensis using Plackett-Burman followed by response surface methodology. IOSRPHR. 2014; 4 (7) 66-75

27- Baoxin Z, Xiangjing W, Wensheng W . Optimization of fermentation medium for enhanced production of milbemycin by a mutant of Streptomyces bingchenggensis $\mathrm{BC}-\mathrm{X}-1$ using response surface methodology. Afr J Biotechnol. 2011; 10 (37): 7225-7235

28- Radhakrishnan M, Gopikrishnan V, Balagurunathan R, Vanajakumar. Effect of critical medium components and culture conditions on antitubercular pigment production from novel Streptomyces sp D25 isolated from Thar desert, Rajasthan. J App Pharm Sci. 2015; 5 (06): 015-019

29- Jose PA, Jebakumar SRD. Successive nonstatistical and statistical approaches for the improved antibiotic activity of rare actinomycete Nonomuraea sp. JAJ18. Biomed Res Int. 2014; 2014: 1-11

30- Wang Y, Fang X, An F, Wang G, Zhang X . Improvement of antibiotic activity of Xenorhabdus bovienii by medium optimization using response surface methodology. Microb Cell Fact. 2011; 10:98

31- Rajeswari P, Jose PA, Amiya R, Jebakumar SRD.Characterization of saltern based Streptomyces sp. and statistical media optimization for its improved antibacterial activity. Front Microbiol. 2015; 5 (Article 753):1-11

32- Abdelwahed NAM, El-Naggar NEA. Repeated batch production of vancomycin using synthetic cotton fibers. Afr J Biotechnol . 2011; 10(57): 12244 -12251

33- Abu-Shady MR, Farid MA, El- Diwany AI, El-Enshasy HA. Studies on rifamycins production by Amycolatopsis mediterranei cells immobilized on glass wool. J Basic Microbiol. 1995; 35(5): 279-284

34- Klein J, Ziehr H . Immobilization of microbial cells by adsorption. J Bacteriol. 1990; 16: $1-16$ 